SLAC-PUB-8101

March 1999

\title{
EMITTANCE DILUTION AND BEAM BREAK UP IN THE JLC/NLC
}

\author{
R.M. Jones $^{\dagger}$, K.L.F. Bane ${ }^{\dagger}$, N.M. Kroll ${ }^{\dagger}$, R.H. Miller ${ }^{\dagger}$, T.O. Raubenheimer ${ }^{\dagger}$ and G.V. Stupakov ${ }^{\dagger}$ \\ $†$ Stanford Linear Accelerator Center, M/S 26, P.O Box 4349, Stanford, CA 94309 \\ ‡University of California, San Diego, La Jolla, CA 92093
}

\begin{abstract}
The passage of a multi-bunch beam through an X-band accelerator with cells and structures misaligned gives rise to wakefields which disrupt succeeding bunches. As a consequence, there is growth in the overall emittance of the final colliding bunches. Here we study this process in the context of a JLC-NLC which employs the recently developed RDDS (Rounded Damped Detuned Structures) accelerators with $120 \mathrm{~s}$ fill time and drives a 90 bunch, $250 \mathrm{~ns}$ bunch train. We investigate cumulative beam break-up (BBU) under various parameter regimes appropriate to the structure which is in the process of being fabricated by KEK and SLAC. We compute the resultant emittance growth by means of analytical expressions and find good agreement with the results we obtain from a particle tracking code. We set misalignment tolerences by specifying allowable emittance growth.
\end{abstract}

Paper presented at the 1999 Particle Accelerator Conference (PAC99)

New York City, New York, USA

March 29-April 2, 1999

This work is supported by Department of Energy grant numbers DE-AC03-76SF00515 ${ }^{\dagger}$ and DE-FG03-93ER40759 
EMITTANCE DILUTION AND BEAM BREAK UP IN THE JLC/NLC

\author{
R.M. Jones ${ }^{\dagger}$, K.L.F. Bane ${ }^{\dagger}$, N.M. Kroll ${ }^{\dagger \dagger}$, R.H. Miller ${ }^{\dagger}$, T.O. Raubenheimer ${ }^{\dagger}$ and G.V. Stupakov ${ }^{\dagger}$ \\ $†$ Stanford Linear Accelerator Center, M/S 26, P.O Box 4349, Stanford, CA 94309 \\ ¥University of California, San Diego, La Jolla, CA 92093
}

\begin{abstract}
The passage of a multi-bunch beam through an X-band accelerator with cells and structures misaligned gives rise to wakefields which disrupt succeeding bunches. As a consequence, there is growth in the overall emittance of the final colliding bunches. Here we study this process in the context of a JLC-NLC which employs the recently developed RDDS (Rounded Damped Detuned Structures) accelerators with $120 \mathrm{~s}$ fill time and drives a 90 bunch, 250 ns bunch train. We investigate cumulative beam break-up (BBU) under various parameter regimes appropriate to the structure which is in the process of being fabricated by KEK and SLAC. We compute the resultant emittance growth by means of analytical expressions and find good agreement with the results we obtain from a particle tracking code. We set misalignment tolerences by specifying allowable emittance growth.
\end{abstract}

\section{INTRODUCTION}

The primary consideration in a linear collider is the luminosity of the final colliding beams. The luminosity is given by: $L=\mathrm{fn}_{1} \mathrm{n}_{2}\left(4 \pi \sigma_{\mathrm{x}} \sigma_{\mathrm{y}}\right)^{-1}$, where $\mathrm{f}$ is the collision frequency, $n_{1}$ and $n_{2}$ are the number of the particles in each of the colliding bunches and the beam dimension in the horizontal (vertical) plane is given by: $\sigma_{\mathrm{x}(\mathrm{y})}=\left(\beta_{\mathrm{x}(\mathrm{y})} \varepsilon_{\mathrm{x}(\mathrm{y})}\right)^{1 / 2}$ Here, $\varepsilon_{\mathrm{x}(\mathrm{y})}$ is the horizontal (vertical) emittance and $\beta_{x(y)}$ is the horizontal (vertical) focussing function. Thus, in order to maximize $L$ it is required to minimize the emittance and maximize the population of each bunch. Also, to increase f, whilst using the RF energy more efficiently, in our design we accelerate a train of 90 bunches, each separated from its neighbor by $2.8 \mathrm{~ns}$. As the highly charged bunches traverse the linac any misalignment in the structure, focussing magnets, or initial offset in the leading bunch, gives rise to wakefields [1] which dilute the emittance and the beam may break up down linac. This BBU can result from short range wakes over the bunch itself, in which the head drives the tail, or from a long range wake, in which trailing bunches are driven by leading ones and a cumulative BBU occurs due to the coupled motion of the bunches. The subject of this paper is stucture tolerance and cumulative BBU and means to avoid it.

\section{BBU DUE TO LONG RANGE WAKES}

To reduce the effect of modes adding together coherently and driving the bunches resonantly off-axis, we utilise
4,720 X-band RDDS structures [1]. The accelerating mode is that of an almost constant gradient structure, but the resulting transverse mode (predominantly dipole in character) is forced to decohere and the wakefield falls off in with a Gaussian profile. As there are a finite number of cells then the wake field eventually recoheres and for this reason, weak damping is provided via four manifolds [1] running along the axis of the accelerator If these wakes can be kept small enough the emittance dilution can be kept to acceptable levels, and this sets a limit on the structure alignment tolerances.

However, we have found that BBU occurs when the wakefield is relatively well-damped and it occurs due to a single mode or small number of modes which constitute the wakefield. One indication as to whether or BBU will occur is provided by $\mathrm{S}_{\sigma}$, the RMS deviation of the sum wake from the mean wake (discussed in section 3 ) and we have conducted extensive investigations into $S_{\sigma}$ as a function of the variation of a small error in the bunch spacing.

For the excellent damping provided by an idealized version of RRDS, the wakefield is insufficient for BBU to occur. The value of $S_{\sigma}$, at the nominal bunch spacing is approximately $0.3 \mathrm{~V} / \mathrm{pC} / \mathrm{mm} / \mathrm{m}$ and this is not expected to give any serious instabilities. However, at $0.2 \%$ increase in the bunch spacing there is a peak in $\mathrm{S}_{\sigma}$ (almost double the value at the nominal bunch spacing) and for this worst case, we track [2] the beam down $10 \mathrm{~km}$ of accelerator structures. We perform a similar tracking of particles for a more realistic structure with HOM couplers terminated with VSWR $\neq 1$ and with the last and first cell of the structure decoupled from the manifold. In this case $\mathrm{S}_{\sigma}$ at the designed bunch spacing is 0.45 $\mathrm{V} / \mathrm{pC} / \mathrm{mm} / \mathrm{m}$ and there is a maximum at approximately $0.7 \%$ away from the nominal bunch spacing, where $S_{\sigma}$ reaches $0.88 \mathrm{~V} / \mathrm{pC} / \mathrm{mm} / \mathrm{mm}$.

For both of these cases we record the emittance growth as the particles progress down the linac, and the vertical space at the end of the linac. The phase space is bounded by the unit circle and all points that lie within this circle are stable. In Fig 1 the idealised RDDS for both the vertical and horizontal planes (the latter being a factor of approximately 100 times larger than the former). In this case little emittance growth is seen to result from the long range wakefields. Similarly, the vertical displacement of the bunches at the end of the linac is small (the maximum displacement is approximately $0.125 \mu \mathrm{m}$ ). Furthermore, a glance at the lowermost curve indicates that all bunches are well contained within the unit circle. Decoupling a 

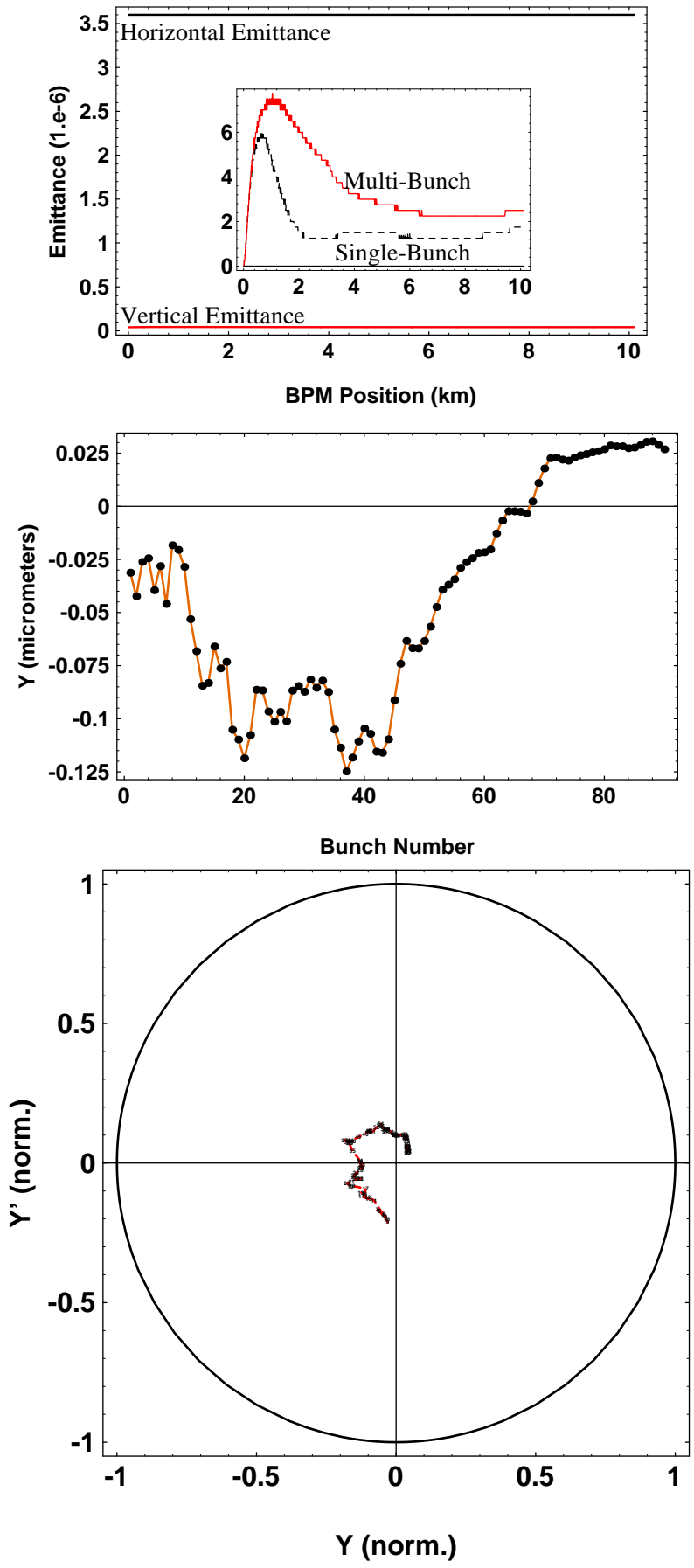

Figure 1: The above shows the result of tracking through $10 \mathrm{~km}$ of accelerator a beam with an initial bunch offset of $1 \mu \mathrm{m}$ or approximately one quarter of $\sigma_{\mathrm{y}}(=3.9 \mu \mathrm{m})$ in the vertical plane and $1 \mu \mathrm{m}$ in the horizontal plane $\left(\sigma_{\mathrm{y}}=\right.$ $35.9 \mu \mathrm{m})$. Uppermost is shown the normalised vertical and horizontal emittance in units of $\mu \mathrm{rad} . \mathrm{m}$ (and inset is indicated the emittance growth as a percentage of the initial emittance at injection to the Xband linacs, for both the single bunch and the multi-bunch cases). In phase space we normalise, $\mathrm{Y}$, the vertical dispacement, with respect to $\left(\beta_{\mathrm{y}} \varepsilon_{\mathrm{y}}\right)^{1 / 2}$ and $\mathrm{Y}^{\prime}$ 'with respect to $\left(\varepsilon_{\mathrm{y}} / \beta_{\mathrm{y}}\right)^{1 / 2}$
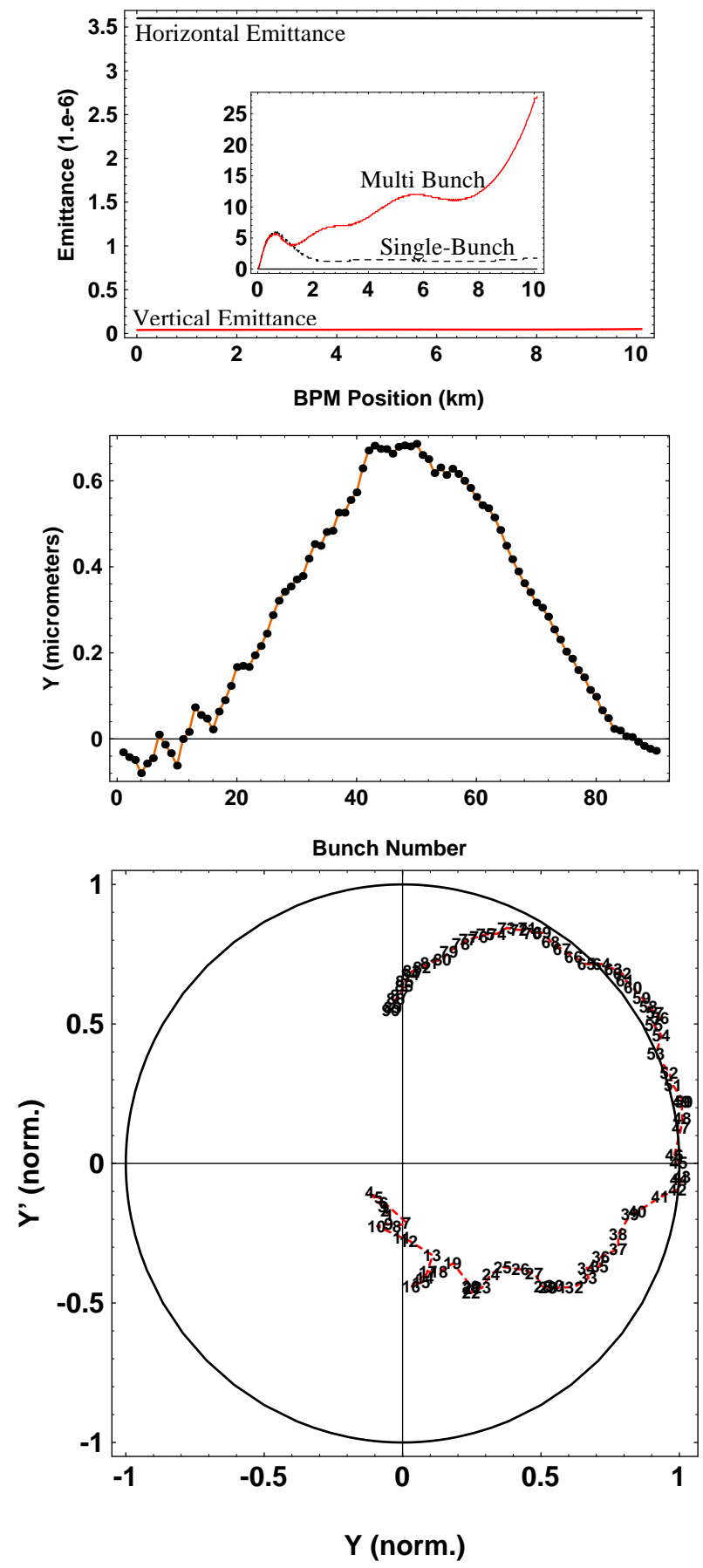

Figure 2: The simulation is similar to that of Fig. 4 except that all of the structures have one cell decoupled and the HOM couplers have realistic terminations, i.e. frequency dependent reflection coefficients corresponding to the transition between circular and rectangular manifold have been included. In both cases, the short range (transverse and longitudinal) wake has been included in the simulations, together with the long range tranverse wakefield. BNS damping of the short range wakefield has been included in the simulations by including a variation in the phase of the R.F. over the linac. 
single cell leads to a quite different situation and, this is shown in Fig 2. Here the emittance grows significantly, to about $28 \%$ larger than the injected value and, as it continues to grow in an exponential fashion, any additional length added to the linac can be expected to lead to further emittance growth. The bunches rapidly are lost from the unit circle boundary in phase space as a cumulative mode of BBU develops within the linac (lower-most curve of Fig 2).

\section{TOLERANCE ON ALIGMENT DUE TO LONG RANGE WAKEFIELDS}

In order to estimate the growth of the projected emittance $\Delta \varepsilon$ of a train of bunches caused by misaligned structure cells we uses the following formula for the expectation value of $\Delta \varepsilon[3]$

$$
\langle\Delta \varepsilon\rangle=\mathrm{r}_{\mathrm{e}}^{2} \mathrm{~N}^{2} \bar{\beta}_{0} \mathrm{~L}_{\mathrm{s}}^{2}\left\langle\Delta \mathrm{S}_{\mathrm{k}}^{2}\right\rangle \frac{1-\left(\gamma_{0} / \gamma_{\mathrm{f}}\right)^{1 / 2}}{\gamma_{0}^{1 / 2} \gamma_{\mathrm{f}}^{3 / 2}}
$$

where $r_{e}$ is the classical electron radius, $\mathrm{N}$ is the number of is the number of particles in the bunch, $\bar{\beta}_{0}$ is the average value of the beta function at the beginning of the linac, $\mathrm{N}_{\mathrm{s}}$ is the number of structures in the linac, $\mathrm{L}_{\mathrm{s}}$ is the length of the structure, $\gamma_{0}$ and $\gamma_{\mathrm{f}}$ are the initial and final relativistic factors of the beam, and $S_{k}$ is the sum wake. The quantity $\mathrm{S}_{\mathrm{k}}$ is defined as a sum of the transverse wakes $\mathrm{w}_{\mathrm{i}}$ generated by all bunches preceding the bunch number $\mathrm{k}$, $\mathrm{S}_{\mathrm{k}}=\sum_{\mathrm{i}=1}^{\mathrm{k}} \mathrm{w}_{\mathrm{k}}$ and $\Delta \mathrm{S}_{\mathrm{k}}$ is the the difference between $\mathrm{S}_{\mathrm{k}}$ and the average value $\langle\mathrm{S}\rangle$, with $\langle\mathrm{S}\rangle=\mathrm{N}_{\mathrm{b}}^{-1} \sum_{\mathrm{k}=1}^{\mathrm{N}_{\mathrm{b}}} \mathrm{S}_{\mathrm{k}}$, where $\mathrm{N}_{\mathrm{b}}$ is the number of bunches. Also, $\mathrm{S}_{\sigma}=\left\langle\Delta \mathrm{S}_{\mathrm{k}}^{2}\right\rangle^{1 / 2}$. Eq. 3.1 is derived assuming a lattice with the beta function smoothly increasing along the linac as $\bar{\beta} \propto \mathrm{E}^{1 / 2}$.

For small misalignments, $\mathrm{w}_{\mathrm{i}}$ is a linear function of cell offsets, $\mathrm{w}_{\mathrm{i}}=\sum_{\mathrm{k}=1}^{\mathrm{N}_{\mathrm{c}}} \mathrm{W}_{\mathrm{is}} \mathrm{y}_{\mathrm{s}}$ which can be found from the solution of Maxwell's equations for the structure. The matrix $\mathrm{W}_{\text {is }}$ for the NLC structure RDDS1 with 206 cells is based on the method [4]. It has a dimension of $\mathrm{N}_{\mathrm{b}} \times 206$. In our calculation we used $\mathrm{N}_{\mathrm{b}}=90$ for bunch spacing 2.8 ns.

By splitting the structure into k pieces of length $1=\mathrm{L}_{\mathrm{s}} / \mathrm{k}$ and randomly misaligning each piece with an rms value of $\Delta$ so that misalignments of different pieces are uncorrelated, we calculated the wake and found the emittance growth of the train of bunches as a function of parameter $\Delta$. Requirement of the emittance growth to be less then $10 \%$ of the nominal vertical emittance of the NLC beam gives a tolerance for the amplitude of the misalignments. This tolerance is shown in Fig. 1 with the following linac parameters: beam final energy $-\mathrm{E}_{\mathrm{f}}=500$ $\mathrm{GeV}$, number of structures in the linac $-\mathrm{N}_{\mathrm{s}}=4720$, and number of particles in the bunch $-\mathrm{N}=1.1 \times 10^{10}$.
To verify the accuracy of the analytical formula (3.1) we also calculated the tolerances using the computer simulation program LIAR . The results of the simulations are also shown in Fig. 3. It is seen that the analytical formula generally agrees rather well with the simulation.

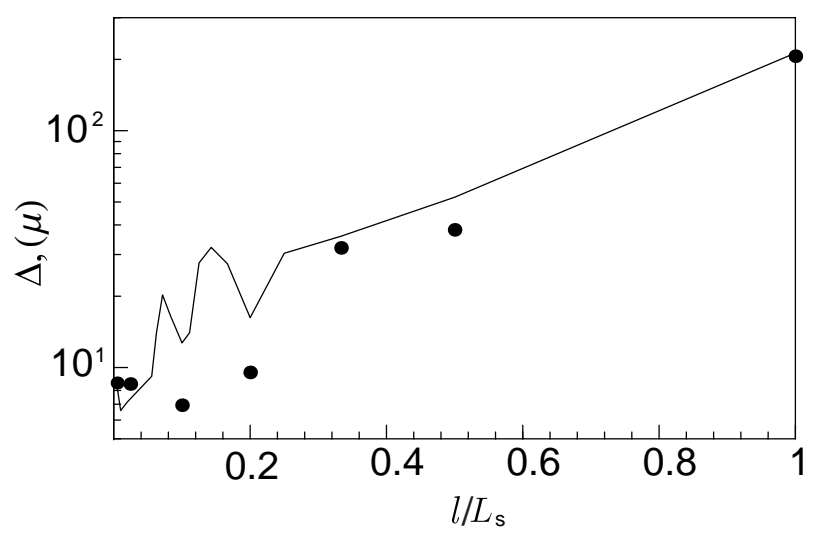

Figure 3: Tolerance $\Delta$ vs. misalignment length 1 in units of the structure length $L_{s^{\prime}}$. The solid curve shows the result of the analytical calculation based on Eq. (3.1); dots are the tolerances calculated using LIAR

Also, we found the tolerance for the structure bow which can be caused by either structure sagging between the support points under the influence of the structure weight, or thermal expansion of the structure. In this calculation, we assume that shape of a bowed structure is given by $\mathrm{y}_{\mathrm{s}}=\mathrm{y}_{0} \sin \left(\pi \mathrm{s} / \mathrm{N}_{\mathrm{s}}\right)$, and that the amplitude $\mathrm{y}_{0}$ is a random number for different structures in the linac. The tolerance for this kind of misalignment is $\Delta=160 \mu \mathrm{m}$

\section{DISCUSSION}

In both scenarios envisaged in Fig. 1 and 2 the worst case of $S_{\sigma}$ is used. In practice the structure will average over these errors and BBU will not necessarily occur in the case of one cell decoupled. However, additional tracking for the case of 4 cells decoupled (as in the present design for RDDS 1 which is in the process of being fabricated [5]) BBU will occur at the nominal bunch spacing. This may be alleviated by direct loading [6] of the final few cells with, for example, $\mathrm{SiC}$ slotted into the last 4 cells.

\section{ACKNOWLEDGMENTS}

This work is supported by Department of Energy grants DE-FG03-93ER40759 ${ }^{\ddagger}$ and DE-AC03-76SF00515 $5^{\dagger}$.

\section{REFERENCES}

[1] R.M. Jones et al, this conference, FRA37

[2] R. Assman et al, 1997, SLAC-PUB AP-103

[3] K.L.F. Bane et al, EPAC94 (also SLAC-PUB 6581)

[4 ] R.M. Jones et al, PAC97 (also SLAC-PUB 7538)

[5 ] J.W. Wang et al, this conference, FRA18

[6] R.M. Jones et al, this conference, FRA38 